11 a 13 de setembro de 2019 - Universidade de Brasília UnB

\title{
ANÁLISE DE MODELOS DE ACEITAÇÃO DE TECNOLOGIA PARA MEDIDORES DE ENERGIA INTELIGENTES
}

Jonathan Gumz (jonathan.gumz@gmail.com) - Universidade Federal de Santa Catarina

Diego Castro Fettermann (․fettermann@ufsc.br) - Universidade Federal de Santa Catarina

\section{RESUMO}

A crescente demanda por energia elétrica e a preocupação com o clima do planeta aumentam a demanda por produção de energia renovável, advinda de diversas fontes de geração, como eólica e solar. Para se aproveitar com eficiência a energia gerada destas fontes é preciso transformar a atual rede elétrica disponível nas cidades em uma rede elétrica inteligente (smart grid), capaz de gerenciar com maior eficiência a energia produzida, armazenada e consumida. Um dos principais fatores para o pleno desenvolvimento desta rede elétrica inteligente é a utilização de medidores de consumo inteligentes pelos consumidores residenciais. Apesar disso, diversos estudos internacionais mencionam a baixa aceitação e engajamentos dos clientes residenciais na utilização destes medidores em suas residências. A partir disso, este trabalho contribui para a discussão relacionando, por meio de uma revisão da literatura, a adequação do uso de duas teorias de aceitação de tecnologia e apontando para uma possível oportunidade existente de se utilizar o Modelo de Aceitação de Tecnologia Baseado em Valor para modelar a aceitação de medidores inteligentes pelos consumidores residenciais, tornando essa aceitação mais fácil e assim utilizando todo o potencial da rede elétrica inteligente.

Palavras chave: Medidor Smart, Rede Smart, Aceitação. 


\section{INTRODUÇÃ̃}

A rede de geração e distribuição elétrica consiste em um sistema complexo projetado para operar de forma integrada, produzindo, transmitindo e entregando energia aos consumidores de forma imediata e simultânea (ELLABAN; ABU-RUB, 2016). Contudo, este sistema elétrico foi projetado para utilizar principalmente combustíveis fósseis, cuja parcela na produção mundial vem diminuindo de maneira consistente e em ritmo acelerado (BP, 2014; REN21, 2018). Atualmente se verifica uma transformação no uso dessas fontes de energia, sendo que a parcela de produção de energia a partir dos denominados recursos renováveis de energia tem aumentado em nível mundial (REN21, 2018). Entretanto, muitas destas fontes de energia renovável, tais como a energia eólica e a solar, apresentam uma produção intermitente, dependente do clima e mais descentralizada, organizada principalmente em pequenas usinas e produtores residenciais (ELLABAN; ABU-RUB, 2016). Apesar da crescente participação na geração de energia a partir das fontes renováveis, a infraestrutura da rede elétrica não foi projetada para o fornecimento de energia com este maior nível de variabilidade, resultando em um sistema menos confiável e mais sujeito a interrupções no fornecimento (ELLABAN; ABURUB, 2016).

A adaptação da rede elétrica atual para incorporar esta maior diversidade de fontes de energia de forma eficiente e racional acontece a partir de sua transformação para uma rede elétrica inteligente (smart grid, em inglês) (HOSSAIN et al., 2016). A rede elétrica inteligente permite uma comunicação bidirecional em tempo real, integrando todo o fornecimento, armazenamento e consumo de energia, por meio de algoritmos inteligentes de gerenciamento de energia, controle automatizado e tecnologias modernas de comunicação, possibilitando maior eficiência, confiabilidade e segurança (ELLABAN; ABU-RUB, 2016). O desenvolvimento de uma rede elétrica inteligente é construído por meio de cinco principais áreas, apresentadas em ordem crescente de importância: Tecnologia, Padrões, Segurança e Privacidade, Tarifas e Regulamentos, Aceitação e Envolvimento do Consumidor (RAHMAN, 2015). Apesar disso, o foco das pesquisas que abordam o desenvolvimento da rede elétrica inteligente tem sido a tecnologia, partindo de um pressuposto que a aceitação e o envolvimento do consumidor final estão garantidos. Essa suposição pode prejudicar severamente a eficiência real da rede elétrica inteligente, dado que o uso racional da energia depende da cooperação do consumidor (ELLABAN; ABU-RUB, 2016; MANIC et al., 2016; ÜRGE-VORSATZ et al, 
2015). Espera-se uma alteração no comportamento do consumidor junto com a transformação da rede elétrica, de uma posição periférica e passiva, para uma posição de consumidor e produtor, cujo comportamento e padrões de uso apresentam efeito sobre todo o sistema da rede elétrica inteligente (PARK; KIM; YONG, 2017). Entretanto, diversos estudos realizados nos Estados Unidos (BUGDEN; STEDMAN, 2019), França (BERTOLDO; POUMADÈRE; RODRIGUES JUNIOR, 2015), Índia (KUMAR, 2019) e Reino Unido (BUCHANAN et al., 2016) indicam diversas restrições dos consumidores em relação a sua participação neste sistema integrado.

Dentro da rede elétrica inteligente, o aparelho físico mais próximo do consumidor final e que exerce maior influência na opinião do consumidor sobre a rede inteligente é o medidor de consumo inteligente (smart meter) (AVANCINI et al., 2019). O medidor inteligente substitui o medidor de consumo tradicional, conhecido como medidor eletromecânico de watt-hora, porque o desenvolvimento da rede inteligente gera a necessidade de novas formas e funções de monitoramento e controle, incompatíveis com o medidor tradicional (SUN et al., 2016). Estes medidores inteligentes apresentam diversas possibilidades de funções e módulos, tais como conexão física e sem fio, tela interativa, medição e cobrança bidirecional, alarmes visuais e sonoros, armazenamento e gerenciamento de dados (AVANCINI et al., 2019). Neste sentido, a familiaridade dos consumidores com o medidor inteligentes e suas funcionalidades é decisiva para o pleno funcionamento da rede elétrica inteligente como um todo (BUGDEN; STEDMAN, 2019), sendo que sua aceitação e envolvimento é considerada essencial para o desenvolvimento total da rede elétrica inteligente (ELLABAN; ABU-RUB, 2016).

A partir disso, este trabalho busca contribuir com a discussão sobre a aceitação de medidores inteligentes pelos consumidores residenciais, com base na literatura, trazendo casos de aplicação de dois modelos para aceitação de tecnologia aplicáveis à rede inteligente. A partir dos resultados apresentados busca-se identificar a melhor forma de compreender e modelar os fatores de afetam a aceitação e envolvimento do consumidor doméstico no uso de medidores inteligentes.

\section{REVISÃO DE LITERATURA}

\subsection{MODELO DE ACEITAÇÃO DE TECNOLOGIA}

O Modelo de Aceitação de tecnologia foi proposto originalmente por Davis (1989) a 
fim de predizer quais fatores apresentariam efeito na aceitação de computadores por usuários. Nesse modelo, a aceitação de uma tecnologia por um usuário é prevista a partir de duas crenças importantes: utilidade e facilidade de uso percebidas pelo usuário. A utilidade percebida é definida como a crença de que usar uma determinada tecnologia aumentará o desempenho do trabalho do usuário. Facilidade de uso é definida como a crença de que usar uma determinada tecnologia será fácil. Por meio de questionários aplicados a usuários de computadores, Davis (1989) conclui que essas duas crenças influenciam a atitude em direção ao uso, e que a atitude em direção ao uso interage com a utilidade percebida para modificar a intenção comportamental de uso por parte do usuário. $\mathrm{O}$ uso propriamente dito é afetado pela intenção comportamental de uso da mesma maneira que a utilidade percebida é influenciada pela facilidade de uso percebida (DAVIS, 1989) (Figura 1)

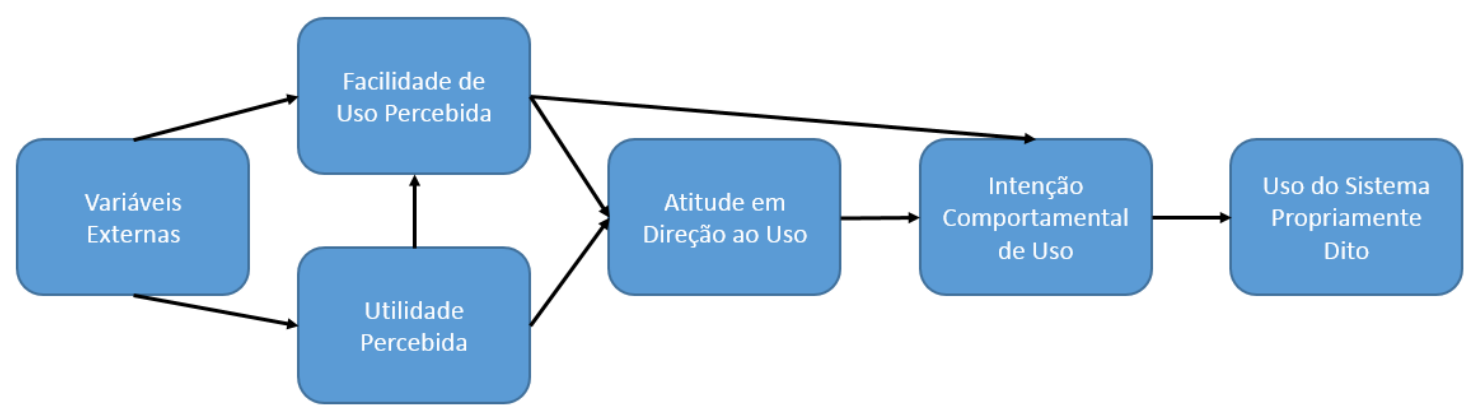

Figura 1: TAM (DAVIS, 1989)

Após a publicação do TAM (DAVIS, 1989), estudos foram conduzidos para modificar e estender a aplicação do modelo em outras situações (GEFEN; KARAHANNA; STRAUB, 2003; KOUFARIS; HAMPTON-SOSA, 2002; PARK; KIM; KIM, 2014; VENKATESH; DAVIS, 2000). O próprio autor publicou o chamado TAM II (VENKATESH; DAVIS, 2000), no qual, além de simplificar o modelo removendo a atitude em direção ao uso, também enfatizou a importância da utilidade percebida, quando comparada à facilidade de uso. A simplificação foi motivada pelos estudos demonstrarem que influência sofrida pela atitude em direção ao uso é semelhante à influência sofrida pela intenção comportamental de uso (VENKATESH; DAVIS, 2000). A importância da utilidade percebida em relação à facilidade de uso percebida é dada pelo fato de a utilidade percebida consistentemente ter mais poder de explicar a intenção de aceitar uma tecnologia do que a facilidade de uso (VENKATESH; DAVIS, 2000). 
Ainda assim, foram registradas críticas ao modelo, principalmente em razão de não refletir todos os pontos de vista dos usuários limitando as variáveis determinantes apenas em utilidade percebida e facilidade de uso percebida (PARK; KIM; KIM, 2014). Outros estudos investigaram a possibilidade de outros fatores para explicar a aceitação dos usuários (GEFEN; KARAHANNA; STRAUB, 2003; KOUFARIS; HAMPTON-SOSA, 2002). A aceitação da rede mundial de computadores foi determinada por cinco fatores: controle percebido, prazer da ação, concentração, além de utilidade percebida e facilidade de uso percebida. (KOUFARIS; HAMPTON-SOSA, 2002). Outro estudo, sobre a aceitação de e-commerce, adicionou a variável confiança ao lado de facilidade de uso percebida e utilidade percebida para explicar a aceitação por parte dos consumidores (GEFEN; KARAHANNA; STRAUB, 2003).

Park, Kim e Kim (2014) sugeriram então uma abordagem de risco percebido integrado ao TAM. Apoiados na ideia de que a aceitação de uma nova tecnologia não é apenas fundamentada em fatores racionais, mas também em fatores irracionais como imagem e emoção (KIM, 2009), eles percorrem uma extensa lista de referências para justificar a ênfase a ser dada para a percepção de risco, integrando assim o modelo em conjunto com a facilidade de uso percebida e a utilidade percebida. É desenvolvido assim, o Modelo de Aceitação de Tecnologia com Risco Integrado (RITAM), conforme a Figura 2.

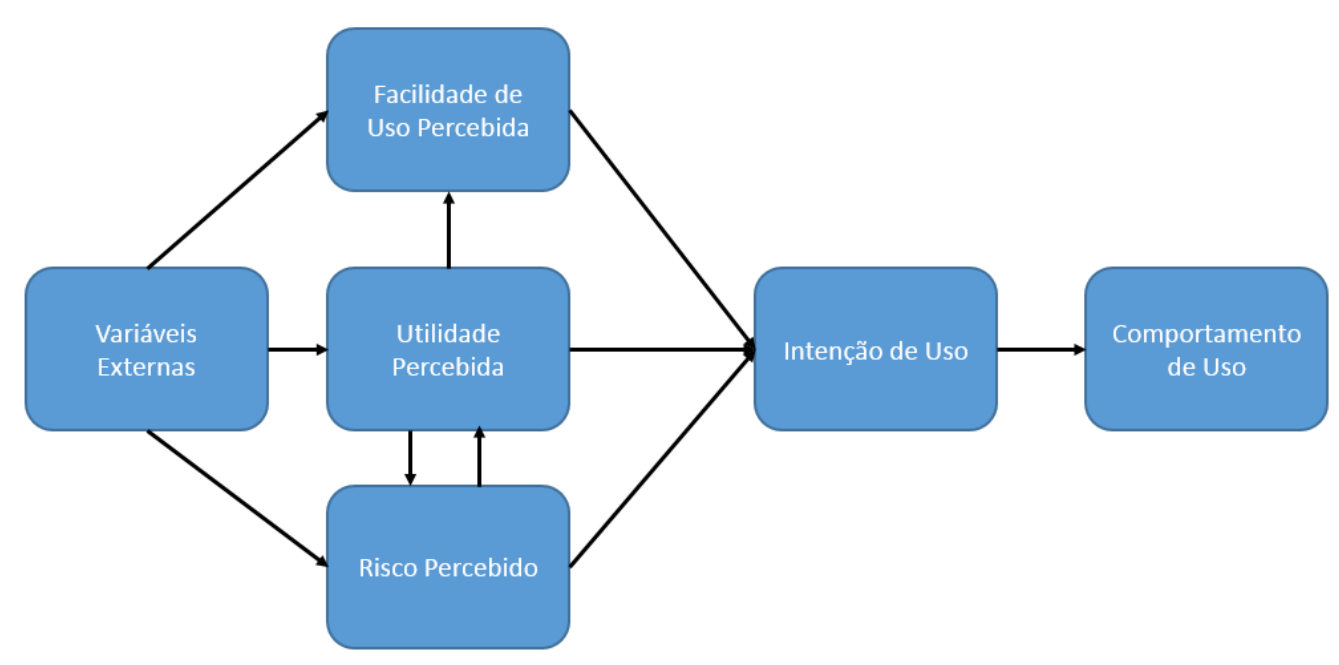

Figura 2: RITAM (PARK; KIM; KIM, 2014)

\subsection{UTILIZAÇÃO DO TAM EM RELAÇÃO À REDE SMART}

São verificados na literatura alguns trabalhos utilizando o TAM para estudar a aceitação 
do consumidor em relação a produtos e serviços da rede inteligente como os de Park, Kim e Kim (2012) e Orillaza, Orillaza e Barra (2014). Park, Kim e Kim (2012) utilizaram o TAM para investigar a aceitação dos consumidores de energia elétrica a respeito da rede elétrica inteligente na Coreia do Sul. Foram adotados como fatores externos: a Percepção de Economia de Energia, na qual adotar a rede inteligente traria uma diminuição do consumo de energia; a Percepção de Ambiente Ecológico (Eco-Enviroment), na qual a rede inteligente ajuda a preservar o meio ambiente; a Percepção de Confiabilidade no Fornecimento de Energia, no qual a rede inteligente não falharia no fornecimento de energia; e a Percepção de Risco de Hacking e Invasão de Privacidade, no qual uma rede inteligente seria propícia para o roubo de informações confidenciais. Utilizando uma metodologia de survey, o resultado da pesquisa sugere que é importante reforçar a percepção de utilidade e facilidade de uso, assim como eliminar preocupações entre os consumidores a respeito de ameaças de segurança da informação pessoal na rede inteligente.

Orillaza, Orillaza e Barra (2014) realizaram dois estudos em relação a adoção de uma tecnologia de medição pré-paga na rede inteligente nas Filipinas. No primeiro estudo, utilizaram o TAM para verificar se os consumidores utilizariam a tecnologia de medição de energia prépaga. |Os resultados indicam que a percepção de facilidade de uso, a percepção de utilidade e a acessibilidade levaria os consumidores a usarem a tecnologia, mas ainda haveria a necessidade de maior envolvimento dos consumidores na rede inteligente. $\mathrm{O}$ segundo estudo aponta algumas preferências que os consumidores teriam para um sistema de medição de energia pré-pago.

Abu et. al. (2014) conduzem uma revisão da literatura do TAM sobre redes inteligentes com publicações entre 1998 e 2013, a fim de encontrar estudos sobre os fatores relacionados ao envolvimento e aceitação dos consumidores à rede inteligente. Os resultados indicam que melhorar as percepções de 'efeito de economia de energia' e 'ajuda na preservação do meio ambiente' apresenta um impacto significativo na utilidade percebida e intenção de uso da rede inteligente (ABU et al., 2014).

Stragier, Hauttekeete e Marez (2010) utilizaram o modelo TAM para verificar as percepções dos consumidores em relação à rede inteligente na Bélgica. Através de uma survey, verificou-se que os consumidores percebem os componentes da rede inteligente como úteis, apesar da complexidade de uso. Os resultados também indicam a preocupação com a segurança da rede e a percepção de que o controle da utilização da energia elétrica não estaria mais com 
o consumidor. Além de tornar os componentes mais fáceis de serem utilizadas, os fabricantes precisam convencer os consumidores de que a rede é segura e que os consumidores teriam o controle sobre a utilização da energia elétrica (STRAGIER, HAUTTEKEETE e MAREZ (2010).

Park, Kim e Kim (2014), após proporem o modelo RITAM, organizaram uma survey com a intenção de validar o modelo e verificar a aceitação dos consumidores em relação à rede elétrica inteligente. Foram utilizadas como variáveis externas a compatibilidade percebida pelo usuário, seu entendimento do sistema, a confiabilidade em relação ao fornecedor de energia, economia na conta de energia, o ambiente ecológico (eco-enviroment), a cyber insegurança, o medo de radiações eletromagnéticas e as preocupações do usuário em relação ao desempenho da rede inteligente. Os resultados indicaram que a compatibilidade e o entendimento percebidos afetam a facilidade de uso. A compatibilidade se refere ao grau em uma nova tecnologia se assemelha à tecnologia atual, em termos de modo de usar e experiência sentida durante o uso (WU; WANG, 2005). O entendimento percebido se refere ao nível de compreensão da nova tecnologia, apresentando relação com sua percepção de complexidade de uso. (PARK; KIM; KIM, 2014). O estudo usa como base surveys aplicadas por Smart Grid Consumer Collaborative (2010 apud PARK; KIM; KIM, 2014), Zpryme (2011 apud PARK; KIM; KIM, 2014) e Park, Kim e Kim (2012) para justificar o uso da confiabilidade percebida em relação ao fornecedor de energia. Os resultados indicam que os respondentes associam uma transformação para rede elétrica inteligente à diminuição de quedas de energia e demais problemas que ocorrem na rede atual. O estudo também justifica o uso de economia na conta de energia percebida através de questionários aplicados pela IBM (2011 apud PARK; KIM; KIM, 2014), ECOALIGN (2010 apud PARK; KIM; KIM, 2014) e Accenture (2011 apud PARK; KIM; KIM, 2014). Essa variável de economia na conta de energia percebida seria a que teria a influência mais forte sobre a utilidade percebida, com os usuários associando a nova rede a uma diminuição do gasto com energia elétrica. As mesmas fontes são usadas para justificar o uso de ambiente ecológico percebido, que seria a percepção de que a nova rede é benéfica para o meio ambiente. As variáveis externas afetando o risco percebido são cyber insegurança percebida, medo de radiações eletromagnéticas percebido e preocupações de performance percebidas, também retirados dos resultados de diversas surveys (BCG, 2010 apud PARK; KIM; KIM, 2014; GLOBALDATA, 2010 apud PARK; KIM; KIM, 2014; IEA, 2011 apud PARK; KIM; KIM, 2014; GREENTECH MEDIA, 2011 apud PARK; KIM; KIM, 2014; 
ZPRYME, 2011 apud PARK; KIM; KIM, 2014). Os resultados do estudo validaram o modelo RITAM e as variáveis externas utilizadas por meio dos resultados obtidos.

Apesar dos estudos relacionando o TAM e o RITAM aos componentes da rede inteligente, talvez esses modelos não consigam explicar a aceitação por completo (ELLABAN; ABU-RUB, 2016). Desde sua elaboração inicial o TAM se propõe a ser aplicado ao uso de tecnologias em um contexto organizacional sem que seja necessário ao usuário ainda arcar com despesas financeiras durante o processo de aceitação (DAVIS, 1989; ELABAN; ABU-RUB, 2016). No contexto da rede inteligente, o consumidor desempenha um papel duplo, de usuário de uma nova tecnologia assim como de cliente de um serviço (ELABAN; ABU-RUB, 2016). Curtius, Künzel e Loock (2012) demonstram que o TAM deveria considerar o valor percebido pelo cliente e que há a necessidade de criação de modelos de negócio para a rede inteligente. Nesse contexto, o Modelo de Adoção de Tecnologia Baseado em Valor pode ser mais adequado que o TAM (ELABAN; ABU-RUB, 2016).

\subsection{MODELO DE ADOÇÃO BASEADO EM VALOR}

O Modelo de Adoção Baseado em Valor (VAM, Value-based Adoption Model, em inglês), modelo proposto por Kim, Chan e Gupta (2007), foi desenvolvido como uma alternativa ao TAM. Originalmente, foi utilizado para investigar a adoção de consumidores do comércio pela Internet, e os autores sustentam que o TAM é um modelo que foi construído para investigar a aceitação de tecnologias tradicionais, como e-mails, compiladores de textos e planilhas por parte de funcionários de organizações (KIM; CHAN; GUPTA, 2007). A consequência disso é de que o custo relacionado a aquisição e uso dessa tecnologia é absorvido pela organização (KIM; CHAN; GUPTA, 2007). Já em casos como o do comércio eletrônico, a adoção é individual e os custos de aquisição e uso são absorvidos pelo próprio consumidor. Assim, não apenas facilidade de uso, utilidade ou percepção de risco seriam suficientes para modelar e predizer a adoção, seria necessário levar em conta também o valor percebido por parte dos usuários da tecnologia que também são consumidores do serviço (KIM; CHAN; GUPTA, 2007). O VAM utiliza o conceito de valor percebido como um saldo entre o que é dado - o sacrifício - e o que é obtido - o benefício - em um produto, definição dada por Dodds e Monroe (1985). (Figura 3) 


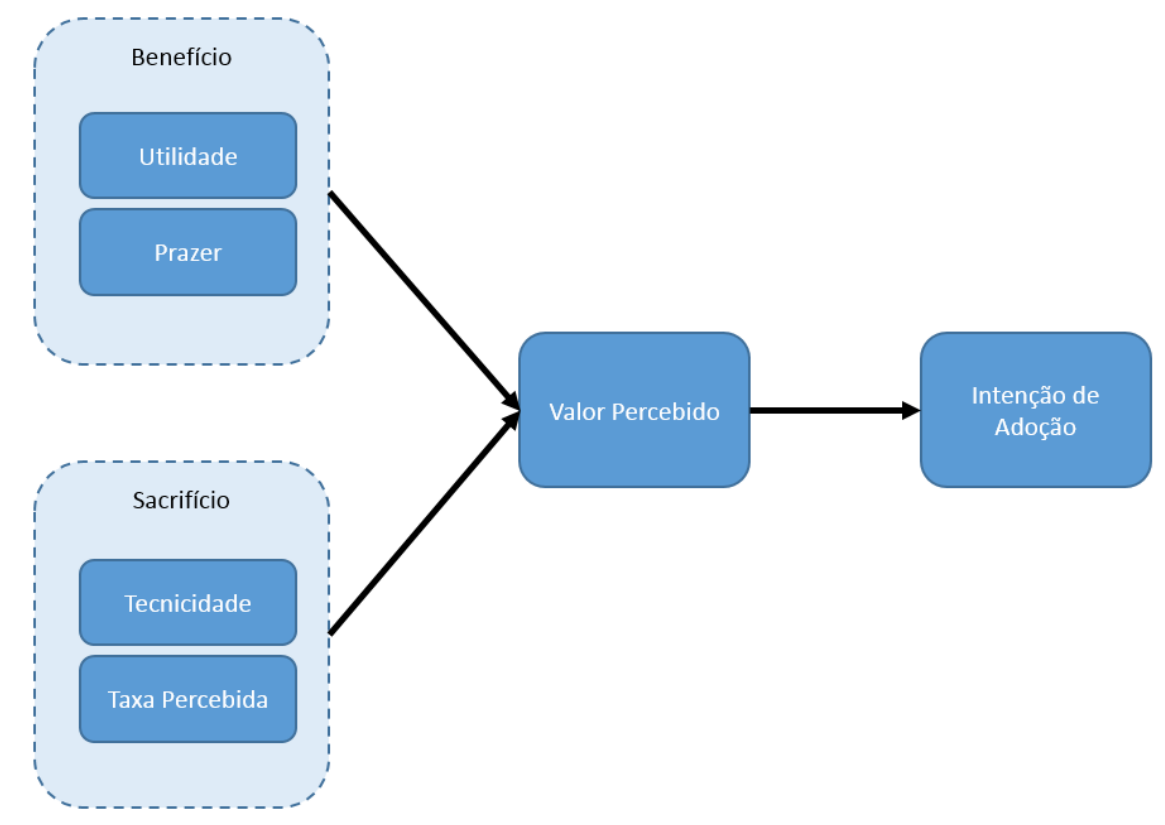

Figura 3: VAM (Value-based Adoption Model) (KIM; CHAN; GUPTA, 2007)

A utilidade e o prazer que compõem o benefício são derivados dos subsistemas extrínsecos e intrínsecos presentes da Teoria da Avaliação Cognitiva (DECI, 1971). De acordo com essa teoria, os subsistemas extrínsecos são motivações referentes ao desempenho de uma atividade para atingir um determinado fim, enquanto os subsistemas intrínsecos de referem às motivações da performance de uma atividade apenas pela realização da atividade em si (DECI, 1971). Dessa forma, o VAM define como utilidade o valor total percebido pelo uso de uma tecnologia, e prazer como a alegria ou sentimento de prazer ao usar uma tecnologia (KIM; CHAN; GUPTA, 2007). Ambos compõem o benefício que afeta positivamente o valor percebido. A tecnicidade e a taxa percebida são derivadas do conceito de que o preço é dividido entre preço monetário e preço não monetário (ZEITHAML, 1988). No VAM, tecnicidade é definido como todo o preço não monetário que deve ser pago por um produto, e a taxa percebida é o preço monetário que o consumidor espera ter que pagar. Tecnicidade e taxa percebida integram o sacrifício que afetam negativamente o valor percebido. Quanto mais positivo é o saldo entre benefício e sacrífico, maior é o valor percebido, que por sua vez afeta positivamente a intenção de adoção (KIM; CHAN; GUPTA, 2007).

\section{RESULTADOS E DISCUSSÃO}

Através de uma análise dos trabalhos não foram identificados estudos que consideram 
o valor percebido pelo usuário na utilização da rede elétrica inteligente. Mesmo assim, se verifica uma oportunidade da utilização do modelo VAM para modelar a aceitação e o envolvimento do usuário à rede elétrica inteligente. Esta oportunidade está indicada na literatura, com trabalhos mostrando itens que são valorizados por consumidores e aumentariam sua intenção de adoção da rede elétrica inteligente (BERTOLDO; POUMADÈRE; RODRIGUES JUNIOR, 2015; BUCHANAN et al., 2016; KUMAR; 2019), e trabalhos adotando o VAM em produtos semelhantes ao dueto tecnologia-serviço dos medidores inteligentes (KIM; CHAN; GUPTA, 2007). Também há uma indicação da mesma oportunidade nas ações dos fornecedores de medidores inteligentes, que planejam adicionar valor percebido aos produtos por meio da inclusão de aplicativos (LANDYS+GYR, 2019).

\section{CONCLUSÃO}

A questão da implantação da rede inteligente é de suma importância em escala global, dada a crescente necessidade do uso de energias renováveis de geração variável e o controle eficiente da geração, armazenamento e consumo da energia elétrica. Apesar do grande desafio da plena utilização da rede inteligente passar pela aceitação dos consumidores residenciais, poucos trabalhos têm investigado os fatores relacionados a essa aceitação. Este trabalho contribui para discussão relacionando duas teorias de aceitação de tecnologia e apontando para uma possível oportunidade existente de se utilizar o VAM para modelar a aceitação de medidores inteligentes pelos consumidores residenciais.

\section{REFERÊNCIAS}

ABU, F. et al. Technology acceptance model (TAM): Empowering smart customer to participate in electricity supply system. Journal of technology management and technopreneurship, [s.l.], v. 2, n. 1, p.85-94, dez. 2014.

AVANCINI, D. B. et al. Energy meters evolution in smart grids: A review. Journal of cleaner production, Elsevier BV, [s.l.], v. 217, p. 702-715, abr. 2019.

BP. BP Energy Outlook 2035. 2014. Disponível em: www.bp.com. Acesso em: 01 maio 2019. 
BERTOLDO, R.; POUMADÈRE, M.; RODRIGUES JUNIOR, L. C. When meters start to talk: The public's encounter with smart meters in France. Energy research \& social science, Elsevier BV, [s.l.], v. 9, p. 146-156, set. 2015.

BUCHANAN, K.; BANKS, N.; PRESTON, I.; RUSSO, R. The British public's perception of the UK smart metering initiative: Threats and opportunities. Energy policy, [s.l.], v. 91, p.8797, abr. 2016. Elsevier BV.

BUGDEN, D.; STEDMAN, R. A synthetic view of acceptance and engagement with smart meters in the United States. Energy research \& social science, Elsevier BV, [s.l.], v. 47, p.137145, jan. 2019.

CURTIUS, H. C.; KÜNZEL, K.; LOOCK, M. Generic customer segments and business models for smart grids. Der markt, Springer Nature, [s.l.], v. 51, n. 2-3, p.63-74, 14 fev. 2012.

DAVIS, F. D. Perceived Usefulness, Perceived Ease of Use, and User Acceptance of Information Technology. Mis quarterly, JSTOR, [s.l.], v. 13, n. 3, p. 319-340, set. 1989.

DECI, E. L. Effects of externally mediated rewards on intrinsic motivation. Journal of personality and social psychology, [s.l.], v. 18, n. 1, p.105-115, 1971. American Psychological Association (APA).

DODDS, W. B.; MONROE, K. B. The Effect of Brand and Price Information on Subjective Product Evaluations. Advances in consumer research, [s.l.], v. 12, n. 1, p.85-90, nov. 1985. Disponível em http://acrwebsite.org/volumes/6364/volumes/v12/NA-12 Acesso em: 26 maio 2019.

ELLABBAN, O.; ABU-RUB, H. Smart grid customers' acceptance and engagement: An overview. Renewable and sustainable energy reviews, Elsevier BV, [s.l.], v. 65, p. 12851298, nov. 2016.

GEFEN, D.; KARAHANNA, E.; STRAUB, D. W. Trust and TAM in Online Shopping: An Integrated Model. Mis quarterly, [s.l.], v. 27, n. 1, p.51-90, 2003. JSTOR.

HOSSAIN, M. S. et al. Role of smart grid in renewable energy: An overview. Renewable and sustainable energy reviews, Elsevier BV, [s.l.], v. 60, p. 1168-1184, jul. 2016.

KIM, H.; CHAN, H. C.; GUPTA, S. Value-based Adoption of Mobile Internet: An empirical investigation. Decision support systems, Elsevier BV, [s.l.], v. 43, n. 1, p. 111-126, fev. 2007. 
KOUFARIS, M.; HAMPTON-SOSA, W. Customer Trust Online: Examining the Role of the Experience with the Web Site. Cis working paper series, New York, v. 5, n. 1, p.1-20, maio 2002.

KUMAR, A. Beyond technical smartness: Rethinking the development and implementation of sociotechnical smart grids in India. Energy research \& social science, Elsevier BV, [s.l.], v. 49, p. 158-168, mar. 2019.

LANDYS+GYR. Apps for Your Meter. 2019. Disponível em: https://eu.landisgyr.com/blogamericas/apps-for-your-meter. Acesso em: 24 maio 2019.

MANIC, M. et al. Building Energy Management Systems: The Age of Intelligent and Adaptive Buildings. Ieee industrial electronics magazine, Institute of Electrical and Electronics Engineers (IEEE), [s.l.], v. 10, n. 1, p. 25-39, mar. 2016.

ORILLAZA, J. R. C.; ORILLAZA, L. B.; BARRA, A. L. Consumer acceptance of prepaid metering and consumer preferences. 2014 Ieee pes asia-pacific power and energy engineering conference (APPEEC), [s.l.], p.1-5, dez. 2014. IEEE.

PARK, C.; KIM, H.; KIM, Y. A study of factors enhancing smart grid consumer engagement. Energy policy, Elsevier BV, [s.l.], v. 72, p.211-218, set. 2014.

PARK, C.; KIM, H.; YONG, T. Dynamic characteristics of smart grid technology acceptance. Energy procedia, [s.l.], v. 128, p.187-193, set. 2017. Elsevier BV.

PARK, C. K.; KIM, H. J.; KIM, Y. S. An empirical study of the smart grid Technology Acceptance Model in Korea. 2012 Ieee international energy conference and exhibition (ENERGYCON), [s.l.], p.110-114, set. 2012. IEEE.

REN21. Renewables 2018 Global Status Report. 2018. Disponível em: http://www.ren21.net/status-of-renewables/global-status-report/. Acesso em: 01 maio 2019. STRAGIER, J.; HAUTTEKEETE, L.; MAREZ, L. Introducing Smart grids in residential contexts: Consumers' perception of smart household appliances. 2010 Ieee conference on innovative technologies for an efficient and reliable electricity supply, [s.l.], p.135-142, set. 2010. IEEE. 
SUN, Q. et al. A Comprehensive Review of Smart Energy Meters in Intelligent Energy Networks. Ieee internet of things journal, Institute of Electrical and Electronics Engineers (IEEE), [s.l.], v. 3, n. 4, p.464-479, ago. 2016.

ÜRGE-VORSATZ, D. et al. Heating and cooling energy trends and drivers in buildings. Renewable and sustainable energy reviews, Elsevier BV, [s.1.], v. 41, p.85-98, jan. 2015.

VENKATESH, V.; DAVIS, F. D. A Theoretical Extension of the Technology Acceptance Model: Four Longitudinal Field Studies. Management science, [s.l.], v. 46, n. 2, p.186-204, fev. 2000. Institute for Operations Research and the Management Sciences (INFORMS).

WU, J.; WANG, S. What drives mobile commerce? Information \& management, [s.l.], v. 42, n. 5, p.719-729, jul. 2005. Elsevier BV.

ZEITHAML, V. A. Consumer Perceptions of Price, Quality, and Value: A Means-End Model and Synthesis of Evidence. Journal of marketing, [s.l.], v. 52, n. 3, p.2-22, jul. 1988. SAGE Publications. 